\title{
Nuclear clusters, bulges and massive black holes in spiral galaxies
}

\author{
Mark A. Hughes ${ }^{1}$, Almudena Alonso-Herrero ${ }^{2}$, John Atkinson ${ }^{1}$, \\ David J. Axon ${ }^{3}$, Dan Batcheldor ${ }^{1}$, C. Marcella Carollo ${ }^{4}$, \\ Alessandro Marconi ${ }^{5}$ and Claudia Scarlata ${ }^{6}$ \\ ${ }^{1}$ Department of Physics, Astronomy and Mathematics, University of Hertfordshire, College \\ Lane, Hatfield, Hertfordshire, AL10 9AB, United Kingdom email: mah@star.herts.ac.uk \\ ${ }^{2}$ Steward Observatory, University of Arizona, 933 N. Cherry Avenue, Tucson, AZ 85721, USA \\ ${ }^{3}$ Department of Physics, RIT, 84 Lomb Memorial Dr., Rochester, NY 14623-5603, USA \\ ${ }^{4}$ Eidgenoessische Technische Hochshule Zuerich, Hoenggerberg HPF G4.3, CH-8092 Zuerich, \\ Switzerland \\ ${ }^{5}$ INAF-Osservatorio Astrofisico di Arcetri, Largo E. Fermi 5, 50125 Firenze, Italy \\ ${ }^{6}$ Space Telescope Science Institute, 3700 San Martin Drive, Baltimore, MD 21218, USA
}

\begin{abstract}
We report on some of the recent work that has come from our Hubble Space Telescope programme to find massive black holes in nearby spiral galaxies. Determining black hole masses from gas kinematics in spiral galaxies is difficult, but results have been reported for NGC 4041 and new results are soon to be reported for NGC 1300 and NGC 2748. Complementary programmes are also looking for the presence of nuclear star clusters in our Space Telescope Imaging Spectrograph (STIS) and archival Near-infrared Camera and Multi-Object Spectrometer (NICMOS) images. We are also currently analysing United Kingdom Infrared Telescope (UKIRT) Fast Track Imager (UFTI) near-infrared images to determine the surface brightness profiles for 37 of the galaxies in the sample.
\end{abstract}

\section{Massive Black Holes in Nearby Spirals}

Massive black holes seem to be a common component of galactic nuclei. Attempts by the community to establish a demography for massive black holes has lead to various correlations between the mass of the black hole and other host galaxy properties (e.g. Ferrarese \& Merritt 2000, Gebhardt et al. 2000, Marconi \& Hunt 2003). However, for all these correlations spiral galaxies are relatively under-represented with respect to elliptical galaxies. To address this problem, and in an attempt to quantify the demography of massive black holes in nearby spiral galaxies, we had an HST/STIS programme (GO:8228, PI:Axon) to determine black hole masses using gas dynamics of the nuclear discs in 54 spirals. Marconi et al. (2003) reported the first black hole mass determination from this programme for NGC 4041, while an atlas of the spectra with reasonable signal to noise are presented in Hughes et al. (2003). Examples of some of the spectra and images, plus colour maps made using archival NICMOS images are shown in figure 1 . The next paper to report black hole masses from the programme is now near completion (Atkinson et al., in prep) and will present results for NGC 1300 and NGC 2748.

\section{Complimentary Work}

While the main emphasis of the program was to find black hole masses, other complimentary work is underway to understand the environments of the centres of these galaxies. One of the principle host-galaxy properties that has been shown to be correlated with black hole mass is stellar velocity dispersion (Ferrarese \& Merritt 2000, Gebhardt 


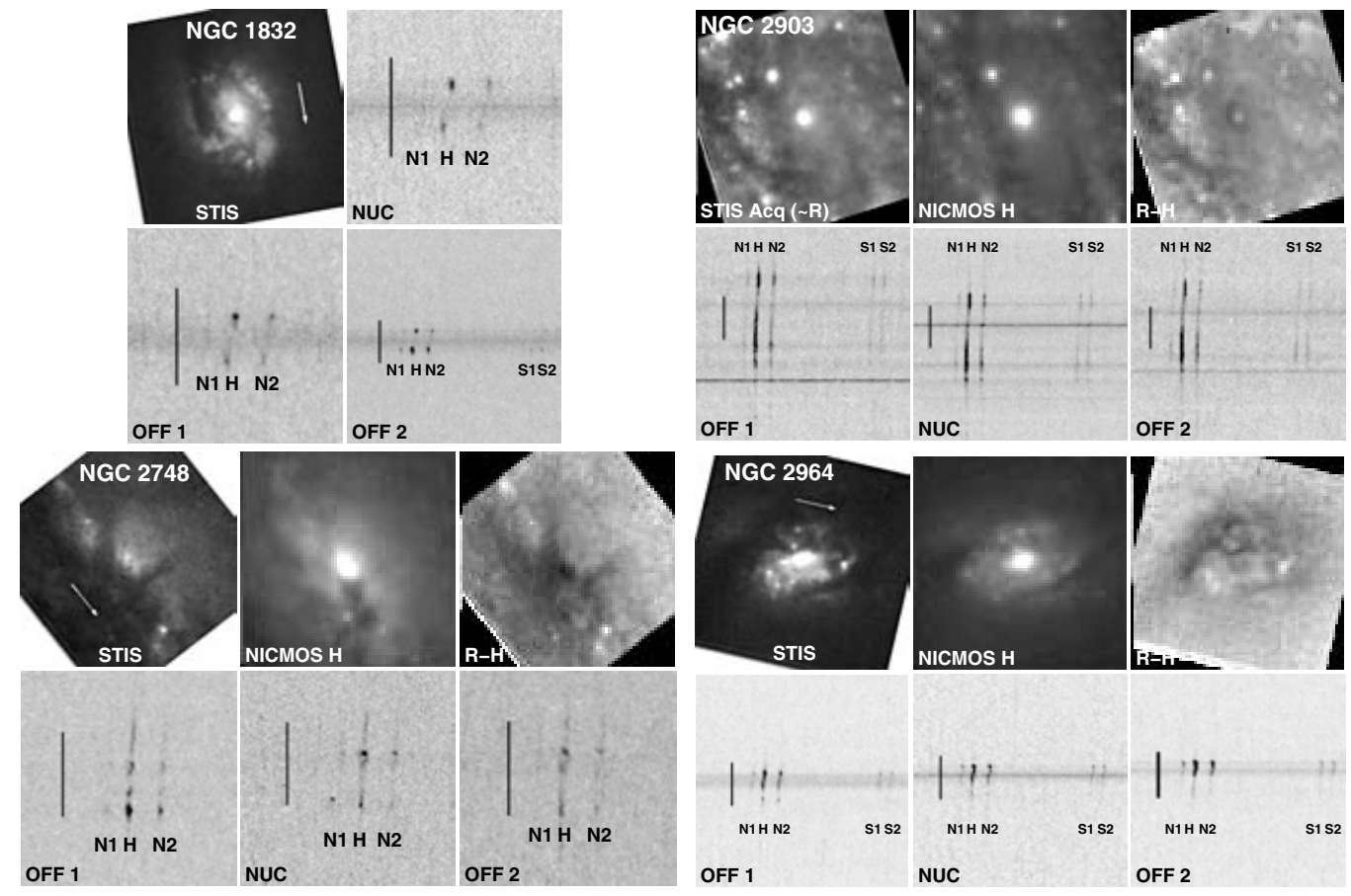

Figure 1. Examples of spectra and images from Hughes et al. (2003). The STIS images ( R-band) and spectra come from our HST program (GO:8228, PI:Axon). Where available, archival NICMOS F160W images ( $\sim \mathrm{H}$-band) were used to produce the $\mathrm{R}-\mathrm{H}$ color maps. The bars indicate a scale of 5 arcseconds and the arrows indicate the direction of the spectroscopic slits. Key: $\mathrm{N} 1=[\mathrm{NII}](6549.9 \AA), \mathrm{H}=\mathrm{H} \alpha(6564.6 \AA)$ and $\mathrm{N} 2=[\mathrm{NII}](6585.3 \AA)$. (reproduced by permission of the AAS).

et al. 2000) and in Batcheldor et al. (in these proceedings) 2-D optical integral-field spectroscopy measurements have been used to determine stellar velocity dispersions.

Imaging has also been used to investigate the nuclear regions. In particular Scarlata et al. (2004) used HST/STIS images to look for the presence of nuclear stellar clusters. Also, Hughes et al (in prep) are using United Kingdom Infra-red Telescope (UKIRT) Fast Track Imager (UFTI) $\mathrm{H}$ and/or K band images to determine the surface brightness profiles (SBP) of 37 of the galaxies from the HST sample. Fits are being made to these surface brightness profiles by combining Sérsic profiles (to represent the bulge) and exponential profiles (to represent the disc component). A correlation between the Sérsic index and black hole mass has been proposed by Graham et al. (2002). If we can obtain reliable profile fits, then our results could be tested against dynamical mass estimates for black hole masses.

\section{References}

Gebhardt, K., et al. 2000, ApJ, 539, L13

Graham, A. W., Erwin, P., Caon, N., \& Trujillo, I. 2002, (astro-ph/0206248)

Ferrarese, L., \& Merritt, D. 2000, ApJ, 539, L9

Hughes, M. A., et al. 2003, AJ, 126, 742

Marconi, A., et al. 2003, ApJ, 586, 868

Marconi, A., \& Hunt, L. K. 2003, ApJ, 589, L21

Scarlata, C., et al. 2004, submitted 